\title{
Erratum to: Non-fecalith-induced appendicitis: etiology, imaging, and pathology
}

\author{
Leonard E. Swischuk ${ }^{1}$ - Dai H. Chung ${ }^{2}$ - Hal K. Hawkins ${ }^{3}$ • \\ Siddharth P. Jadhav ${ }^{4} \cdot$ Ravi Radhakrishnan ${ }^{5}$
}

Published online: 16 April 2016

(C) American Society of Emergency Radiology 2016

Erratum to: Emerg Radiol (2015) 22:643-649

DOI: 10.1007/s10140-015-1338-1

The original version of this article contained errors.

The legends of Figs. 3 and 4 are correct; however, their corresponding images are not. The image shown in Fig. 3 should be for Fig. 4; and the image in Fig. 4 should be for Fig. 3.

Also, some words were missing in the last line of Fig. 3 legend. The last sentence should read "Note the prominent appendiceal tip (arrows) in this patient with early appendiceal tip involvement secondary to lymphoid hyperplasia. This patient also recovered."

The online version of the original article can be found at http://dx.doi.org/ 10.1007/s10140-015-1338-1.

Leonard E. Swischuk

lswischu@utmb.edu

1 Department of Radiology and Pediatrics, The University of Texas Medical Branch, Galveston, TX, USA

2 Department of Pediatric Surgery, Monroe Carell Jr. Children's Hospital at Vanderbilt, Nashville, TN, USA

3 Department of Pathology, The University of Texas Medical Branch, Galveston, TX, USA

4 Department of Radiology, Texas Children's Hospital, Houston, TX, USA

5 Department of Surgery-General Surgery, The University of Texas Medical Branch, Galveston, TX, USA 


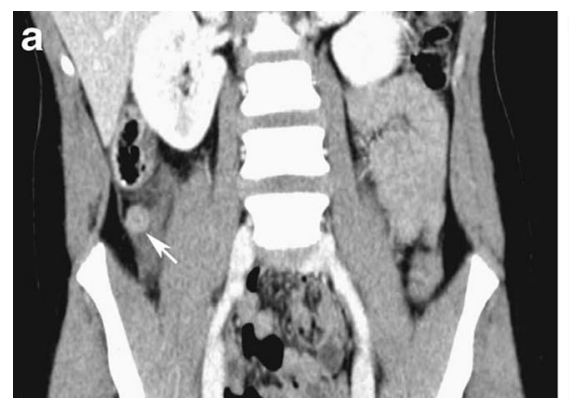

Fig. 3 Prominent tip: reversal offindings. a CT: axial image. This patienthad acute right lower quadrant pain. The appendix (arrow) was identified and the tip appeared a little fuzzy. In addition, there was some early periappendiceal fat stranding. b CT: sagittal reconstruction. Note the swollen appendix which is hyperintense with a prominent, more swollen, and bulbous tip (upper arrow). These findings would be consistent with early viral appendicitis possibly going on to purulent appendicitis. This
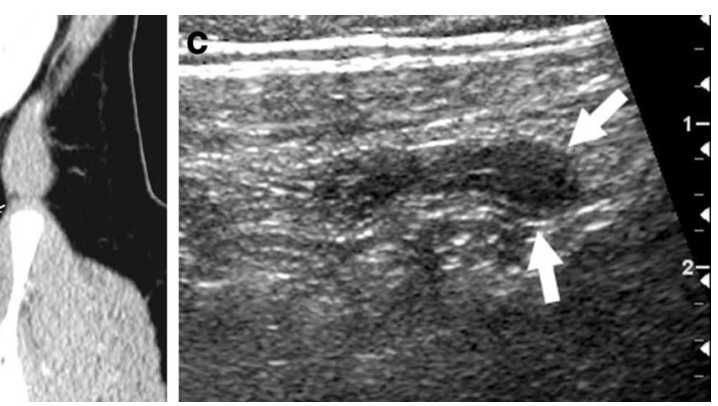

patient was assessed by surgery and thought not to have a surgical abdomen. He was observed for $23 \mathrm{~h}$, and by the end of that time was eating and ready to go home. c Ultrasound findings. Note the prominent appendiceal tip (arrows) in this patient with early appendiceal tip involvement secondary to lymphoid hyperplasia. This patient also recovered
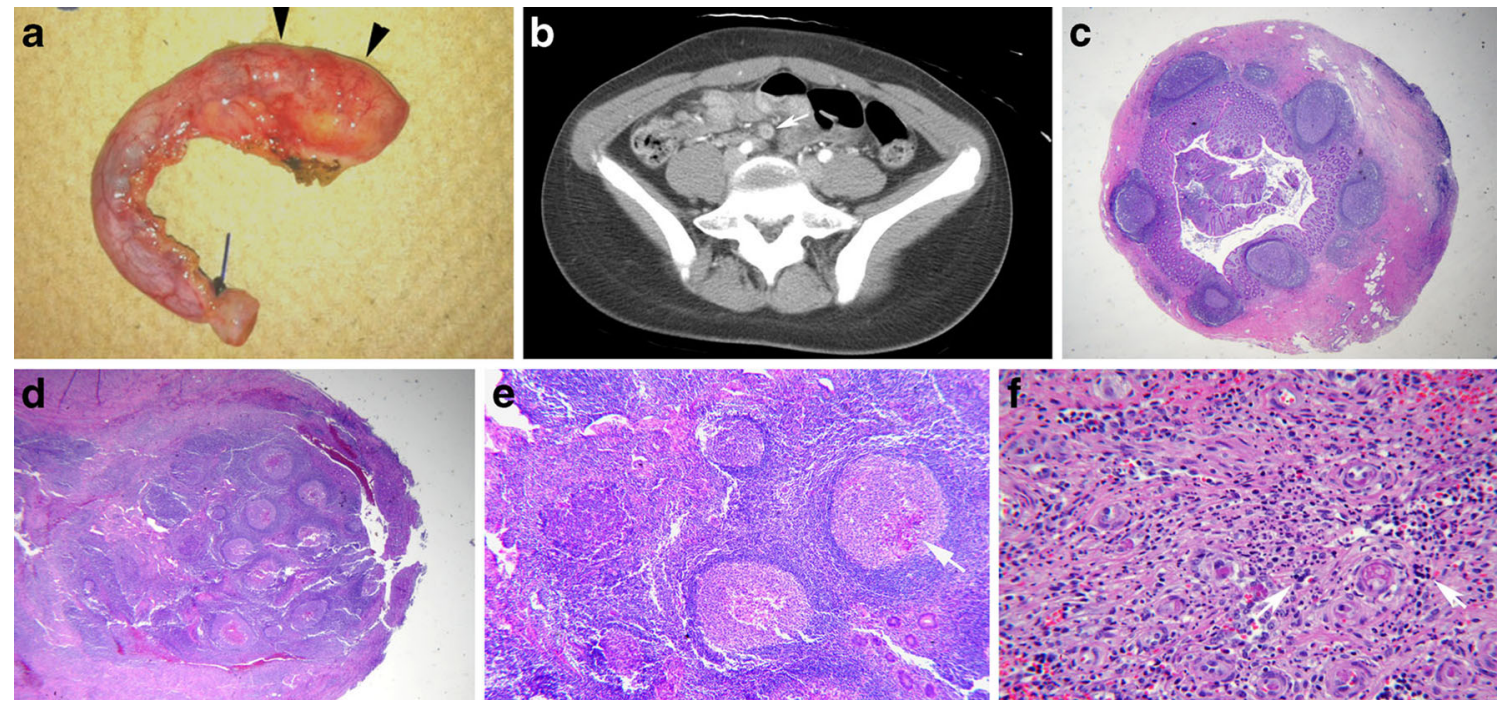

Fig. 4 Pink appendix with bulbous tip. a Note a typical pink appendix but also note that the tip (arrowheads) is swollen, more bulbous, and larger. b Axial CT study in this patient demonstrates the appendiceal tip (arrow) to be a little indistinct and fuzzy consistent with early inflammation. c Low power cross-section histologic image of the proximal appendix. Note the clean, well-formed purple germinal centers

surrounding the lumen. d Low-power longitudinal section of the tip. Again, note numerous purple germinal centers but that some of them appear to be undergoing ischemic/hemorrhagic disorganization. e Highpower view of the germinal centers demonstrates early hemorrhage (arrow). f Another high-power view of the germinal centers shows early infiltration by neutrophils (arrows) 